\title{
Antidepressant drugs in the treatment of anxiety disorders
}

\author{
Allan Scott, Alan Davidson \& Karen Palmer
}

In March 1997 APT published two reviews of the use of benzodiazepines, buspirone, beta-blocking drugs and antidepressants in the treatment of anxiety disorders (Cowen, 1997; Tyrer, 1997). These were followed by a paper on the practical pharmacotherapy of anxiety (Nutt \& Bell, 1997). The present review was originally prompted for several reasons. A number of large-scale investigations of the use of antidepressants in anxiety disorders have been completed since those papers were published. Indeed, several antidepressant drugs have since been licensed to treat anxiety disorders, and more applications are being considered. El-Khayat \& Baldwin (1998) found that the prescription of antipsychotic drugs for anxiety disorders was widespread, but concluded there was no methodologically sound evidence to support their prescription. They suggested that this use of antipsychotic drugs reflected the fears of practitioners about the risks associated with benzodiazepines. There is no reason why the prescription of antidepressant drugs should arouse such fears, and it seemed timely to produce an up-to-date review of their efficacy in the treatment of anxiety disorders. This view was reinforced while this manuscript was in preparation, when the Committee on Safety of Medicines issued a statement in December 2000 that restricted the indications for the prescription of thioridazine because of concerns about rare but serious cardiotoxicity; thioridazine was no longer to be indicated for the treatment of anxiety or psychomotor agitation.

The pharmacotherapy of obsessive-compulsive disorder (Fineberg, 1999) and post-traumatic stress disorder has recently been reviewed in APT (Turner, 2000) and will not be considered further here. Exposure therapy is the treatment of choice for specific phobias (Nutt \& Bell, 1997), and likewise their treatment will not be considered further here. The controversies that affect the context of the present review are listed in Box 1.

\section{Method}

The natural history of anxiety disorders is typically of a fluctuating severity over time, and the earliest controlled trials of drug treatment found a substantial improvement associated with sham or placebo treatment. The major aim of the present review was

Box 1 Controversies surrounding the pharmacotherapy of anxiety disorders

Can anxiety disorders and depressive disorders be reliably distinguished, or do they lie on a single continuum of affective disorder?

Is the pathophysiology of anxiety disorders such as panic disorder now better understood than that of depressive disorders?

Are drug treatments in general underutilised in anxiety disorders?

Have the risks of treatment with benzodiazepine drugs been overemphasised?

Are alternatives such as antipsychotic drugs and beta-blockers consequently overutilised?

Are antidepressant drugs underutilised?

Allan Scott is a consultant psychiatrist and honorary senior lecturer, Alan Davidson is a specialist registrar in psychiatry and Karen Palmer is a staff grade psychiatrist. The authors undertook this project to inform the operational policy of the primary care liaison team in the north-east sector of the general adult psychiatric service associated with the Andrew Duncan Clinic, Royal Edinburgh Hospital (correspondence: Allan Scott, Andrew Duncan Clinic, Royal Edinburgh Hospital, Edinburgh EH10 5HF). 
therefore to identify randomised controlled studies that had included a placebo treatment; moreover, the studies had to have been published and subject to peer review. Studies that included random allocation to a variety of fixed doses were of particular interest because the relationships of prescribed dose to efficacy and tolerability can be assessed. The final date for inclusion was 1 June 2000. Electronic literature searches were conducted on Medline, Embase and the Cochrane Controlled Trials Register. The reference lists of included studies were also searched. Manufacturers of available antidepressant drugs were identified from the British National Formulary (British Medical Association \& Royal Pharmaceutical Society, March 2000) and each was written to on 1 March 2000 and asked to nominate any possible studies of relevance. Anxiety can be symptomatic of a depressive illness and antidepressant drugs may effectively treat such symptoms because they successfully treat the underlying depressive illness. Several antidepressants are now licensed for the treatment of anxiety associated with depressive illness, but the aim of the present study was to review the evidence for their efficacy in primary anxiety disorders. Further details of the search strategy are available from the A.S. upon request.

\section{Panic disorder}

It makes historical sense to start with panic disorder because it was almost 40 years ago that Klein first demonstrated the efficacy of imipramine in reducing the frequency of panic attacks.

\section{Tricyclic antidepressant drugs}

It is well established that imipramine is efficacious in the treatment of panic disorder with or without agoraphobia (Cowen, 1997). It typically takes 4 weeks for the antipanic and antiphobic effects to develop significantly and the maximum effect may not be seen for up to 12 weeks. Paradoxically, there may be an exacerbation of anxiety symptoms in the earliest stages of treatment and for this reason an initial dose of only half a $25 \mathrm{mg}$ tablet daily is suggested. Over the years there have been suggestions that panic disorder can respond dramatically to very low doses of imipramine, but this has not been supported by placebo-controlled fixed-dose studies. It may be necessary to gradually increase the prescribed dose to the equivalent of a little over $2 \mathrm{mg} / \mathrm{kg} /$ day for the optimal effect.
It has been suggested that there is little reason that other tricyclic antidepressant drugs will work any less well in panic disorder than imipramine does. While there is insufficient evidence to confidently support or reject this suggestion, there is some evidence to suggest that tricyclic antidepressants are not equally efficacious and that this may be explained by differences in their potency for the inhibition of the reuptake of either noradrenaline or serotonin. Desipramine predominantly inhibits the reuptake of noradrenaline. Its efficacy was assessed over 12 weeks in 56 subjects who met the DSM-IIIR (American Psychiatric Association, 1987) criteria for panic disorder with or without agoraphobia, but who were free of major depression. Desipramine at a dose of up to $200 \mathrm{mg}$ /day was not more efficacious than placebo in terms of the proportion of subjects who were free from panic attacks after treatment (Lydiard et al, 1993). Other outcome measures did favour active treatment and it may have been that a larger study would have found a statistically significant advantage for active treatment. The study may also have been compromised by the substantial difference in discontinuation between active and placebo treatment; $93 \%$ of the subjects randomised to desipramine completed the 12-week study, but only $61 \%$ of the subjects randomised to placebo treatment did. Maprotiline is another selective inhibitor of the reuptake of noradrenaline; its efficacy has not been compared with that of placebo, but treatment for 6 weeks, including 4 weeks at full dose, did not reduce the frequency of panic attacks in 24 out-patients who met DSM-III (American Psychiatric Association, 1980) criteria for panic disorder with or without severe phobic avoidance (den Boer \& Westenberg, 1988). In contrast, clomipramine is a much more potent inhibitor of the reuptake of serotonin. Modigh et al (1992) reported the findings of a study in which 68 subjects who met the DSM-III criteria for panic disorder with or without agoraphobia, but not major depression, were randomised to 12 weeks of treatment with placebo, imipramine or clomipramine. The maximum permissible dose of antidepressant drug was $250 \mathrm{mg} /$ day; the average maximal dose was $124 \mathrm{mg} /$ day for imipramine and $109 \mathrm{mg} /$ day for clomipramine. Clomipramine led to a significantly greater reduction in the number of panic attacks and anticipatory anxiety than either placebo or imipramine.

It would be wrong to overemphasise the findings of single studies, but there is evidence that a tricyclic antidepressant that is a potent inhibitor of the reuptake of serotonin (clomipramine) may be particularly efficacious in panic disorder. In contrast, there are doubts about the efficacy of tricyclic antidepressants that are selective for the inhibition of the reuptake of noradrenaline. 


\section{Monoamine oxidase inhibitors}

There have been placebo-controlled studies of the efficacy of irreversible monoamine oxidase inhibitors (MAOIs) in agoraphobia and/or social phobias, but these pre-dated the introduction of panic disorder in DSM-III (American Psychiatric Association, 1980). Moreover, they did not report the effect of treatment on the frequency or severity of panic attacks themselves. Nutt \& Bell (1997) suggested phenelzine only as a possible second-line treatment in patients who had already failed to recover with another antidepressant drug.

Moclobemide and brofaromine are reversible and selective inhibitors of monoamine oxidase type A. They are unlike the irreversible inhibitors in that they are not associated with the potentially fatal drug-drug interactions and intolerance of tyraminecontaining foodstuffs. Both drugs have been investigated in the treatment of anxiety disorders, but only moclobemide is commercially available.

One placebo-controlled study of the efficacy of moclobemide was found and this reported that moclobemide ( $600 \mathrm{mg} /$ day) was no more efficacious than placebo in reducing agoraphobic avoidance over 8 weeks (Loerch et al, 1999). The study lacked statistical power because the direct comparison between placebo and active treatment was conducted in only 25 patients who suffered from DSM-III-R criteria panic disorder with agoraphobia (American Psychiatric Association, 1987). There have been two international, multi-centre studies in which the efficacy of moclobemide has been compared with either fluoxetine (Tiller et al, 1999) or clomipramine (Kruger \& Dahl, 1999) over 8 weeks. In the first comparison, the proportions of patients who were panic-free among those who persevered with treatment were $63 \%$ for moclobemide and $70 \%$ for fluoxetine. In the second comparison, the proportions of patients who were panic-free among those who persevered with treatment were $49 \%$ for moclobemide and $53 \%$ for clomipramine. The difference was not statistically significant in either case. The possibility of inefficient dosing with the comparator drug is relevant for both studies. The average maximal dose of fluoxetine was approximately $20 \mathrm{mg} /$ day, a dose that is not efficacious (see below). The initial prescribed dose of clomipramine was $10 \mathrm{mg} /$ day and only reached $75 \mathrm{mg} /$ day after day 19 of the study. The efficacy of moclobemide has not been clearly established.

\section{Selective serotonin reuptake inhibitors}

Most of the recent research in the drug treatment of panic disorder has been conducted with selective serotonin reuptake inhibitors (SSRIs) (see den Boer et al, 2000). Some reviewers have concluded that SSRIs as a group are efficacious in the treatment of panic disorder and that they should be considered as first-line treatment because they are more tolerable and safer than traditional tricyclic antidepressants. Further research may yet support these broad conclusions, but several caveats can be made based on the evidence to date. Only two of the available SSRIs have been assessed against placebo treatment in fixed-dose studies. Sertraline at a dose of between 50 and $200 \mathrm{mg} /$ day was found to be more efficacious than placebo treatment. Not all the placebo-controlled studies of fluvoxamine have found it to be more efficacious than placebo treatment; the studies with positive findings used average maximal doses above $200 \mathrm{mg} /$ day. The minimum efficacious dose for paroxetine was found to be $40 \mathrm{mg} /$ day, and the optimal dose of citalopram was found to be $20-30 \mathrm{mg} /$ day.

Fluoxetine was the most successful branded SSRI and is likely to be even more widely used now that a generic preparation is available. Unfortunately, it has yet to be shown that fluoxetine is unequivocally efficacious in the treatment of panic disorder. A study of 243 patients who met DSM-III-R criteria for panic disorder (with Hamilton Depression Rating Scale scores less than 23; Hamilton, 1960) were randomly allocated to placebo treatment or treatment with fluoxetine $(10 \mathrm{mg} /$ day or $20 \mathrm{mg}$ / day) and no significant difference between either of the active treatments and placebo in the proportion of patients who were free of panic attacks after 10 weeks was found. There were, however, statistically significant differences on other measures of anxiety that favoured active treatment (Michelson et al, 1998).

\section{Other antidepressants}

A placebo-controlled study of venlafaxine was found, but it was too small to draw any firm conclusions. No placebo-controlled studies of trazodone were identified, but the compound was used as a comparator to treatment with imipramine and alprazolam (Charney et al, 1986). The drug was so poorly tolerated that only four of 27 patients randomised to trazodone completed 8 weeks of the trial, and only two patients were eventually considered as recovered. This controlled comparison is mentioned here because of the traditional view that antidepressant drugs with marked sedative effects may be particularly useful in anxious patients; trazodone's apparent lack of efficacy in panic disorder challenges this traditional view. 


\section{Clomipramine and paroxetine compared}

The efficacy and tolerability of paroxetine and clomipramine were compared in a multi-centre study based in 13 countries and funded by the manufacturer of paroxetine (Lucrubier et al, 1997). Threehundred and sixty-seven out-patients who met the DSM-III-R criteria for panic disorder with or without agoraphobia, but not major depression, were randomised to one of the active treatments or placebo. About $70 \%$ of patients randomised to either active treatment completed 12 weeks of treatment. Clomipramine and paroxetine were equally efficacious, in that approximately $50 \%$ of patients were free of panics by the end of treatment, whereas only about $30 \%$ of patients treated with placebo were free of panics. The active treatments were also equally efficacious and superior to placebo treatment in reducing anticipatory anxiety and phobic avoidance. The fall in the frequency of panic attacks in the earlier weeks of the study was most marked for paroxetine. It is not clear to what extent dosing inefficiencies may have contributed to this observation; it was possible to prescribe paroxetine up to a dose of $60 \mathrm{mg} /$ day, whereas the maximum permissible dose of clomipramine was $150 \mathrm{mg} /$ day. Treatment discontinuation because of adverse effects was observed in $7.3 \%$ of the patients prescribed paroxetine and $14.9 \%$ of the patients prescribed clomipramine; this difference was not statistically significant.

\section{Continuation treatment}

Only two published studies in which the longerterm efficacy of antidepressant drug treatment alone was assessed by disguised random allocation of recovered patients to placebo treatment or continuation drug treatment were found. Michelson et al (1999) studied patients who had markedly or moderately improved after 10 weeks' treatment with fluoxetine (10-20 mg daily). The findings of this study are difficult to interpret because this dose of fluoxetine is not efficacious (see above); it cannot be assumed that the patients who improved markedly with such treatment are representative of the generality of patients who suffer from panic disorder. A methodologically sound study was conducted by Mavissakalian \& Perel (1999). Subjects who fulfilled the DSM-III-R criteria for panic disorder with agoraphobia, but not major depression with melancholia, were treated for 24 weeks with imipramine $(2.25 \mathrm{mg} /$ $\mathrm{kg} /$ day); 59 subjects completed treatment and were in stable remission after 24 weeks. Of these subjects, 56 were randomly allocated to either continuation treatment or gradual reduction of imipramine over 3 weeks in a double-blind study. The proportion of patients who relapsed among those allocated continuation treatment for 12 months was $3 \%$ compared to $37 \%$ in those randomised to placebo treatment, a highly statistically significant difference. These findings would certainly support the efficacy of continuation treatment with imipramine for up to 12 months after recovery from panic disorder with agoraphobia. The extent to which these findings can be generalised to less severe types of panic disorder is not known.

\section{Comparisons with psychological treatments}

Several psychological treatments are known to be efficacious in the treatment of panic disorder or panic disorder with agoraphobia; these include applied relaxation, psychological panic management, cognitive-behavioural therapy (CBT) and exposure therapy. A proper discussion of the relative merits of psychological and pharmacological treatments is beyond the scope of the present review, but some general comments on the literature may be of relevance. There has always been interest in which treatment or combination of treatments is most efficacious in the short-term, but recently a belief has emerged that psychological treatments may be more efficacious in the longer term by reducing disability and the risk of relapse. The available evidence is difficult to assess because most controlled comparisons have lacked statistical power because of small samples, and the inclusion criteria have varied among studies. In contrast, Barlow et al (2000) published the findings from a comparison of CBT, imipramine and their combination in the treatment of 312 subjects who met the DSM-III-R criteria for panic disorder with or without mild agoraphobia at four anxiety research clinics. Twenty-seven per cent of the subjects suffered from major depression. Both imipramine and CBT were more efficacious than placebo treatment during the acute phase, but the combination was no more efficacious. Among the subjects who recovered, imipramine produced a response of 'higher quality'. Six months after the end of treatment, the proportion of subjects who maintained their recovery was $96 \%$ for those who had recovered with CBT alone and only $75 \%$ of those who had recovered with imipramine alone, a statistically significant difference; data are not reported for the combination. Psychological and 
pharmacological treatments in both continuation and prophylactic phases of treatment clearly merit further study.

\section{Social phobia}

Social phobia, also known as social anxiety disorder, has been a topic of debate. Some commentators have noted that it is the most prevalent anxiety disorder in community surveys and that it may be a risk factor for the development of other psychiatric disorders such as agoraphobia, major depression or substance misuse. Yet the disorder was not recognised in the International Classification of Disease until 1992 (World Health Organization, 1992), and most sufferers identified in community surveys have not been in contact with specialist psychiatric services.

\section{Tricyclic antidepressants}

No placebo-controlled studies were found. A small uncontrolled study found that more than one-third of patients gave up treatment with imipramine within 8 weeks because of adverse effects and among those who completed treatment only $22 \%$ were substantially improved (Simpson et al, 1998).

\section{Monoamine oxidase inhibitors}

Among the irreversible inhibitors, phenelzine is of proven efficacy; it has been shown to lead to substantial reductions in social fear, social avoidance and performance fear over 8 weeks (see Walker \& Kjernisted, 2000). No fixed-dose study has been conducted and average maximal doses have been in excess of $65 \mathrm{mg} /$ day. The extent of clinical improvement seen with phenelzine has been less dramatic in the more recent studies. Why this should be is not known, but one possibility is that the more recent studies, unlike the first placebo-controlled study, have definitely excluded patients with coexisting major depression.

Moclobemide and brofaromine have also been investigated, although only moclobemide is commercially available (see Walker \& Kjernisted, 2000). The first placebo-controlled study found moclobemide at a dose of $600 \mathrm{mg}$ / day more efficacious than placebo and comparable in efficacy to phenelzine. These findings have not been consistently confirmed. A fixed-dose study found that moclobemide at a dose of up to $900 \mathrm{mg} /$ day over 12 weeks was no more efficacious than placebo treatment in a multicentre study that included 523 subjects who met the
DSM-III-R criteria for social phobia (Noyes et al, 1997).

\section{Selective serotonin reuptake inhibitors}

All five SSRIs available in the UK have been studied in the treatment of social phobia (see Walker \& Kjernisted, 2000), but placebo-controlled studies were identified only for fluvoxamine, paroxetine and sertraline. Each of these has been shown to be more efficacious than placebo treatment, although no fixed-dosed studies have been conducted. The doses at which efficacy was demonstrated were $150 \mathrm{mg} /$ day for fluvoxamine, $20-50 \mathrm{mg}$ /day for paroxetine and 50-200 mg/day for sertraline. By far the largest body of evidence concerned paroxetine. Baldwin et al (1999) reported the findings from a multi-centre European and South African study of 290 subjects who met DSM-IV (American Psychiatric Association, 1994) criteria for social phobia; $66 \%$ of the subjects treated with paroxetine for 12 weeks were rated as significantly improved compared to $32 \%$ of the subjects treated with placebo.

\section{Generalised anxiety disorder}

Klein himself doubted the efficacy of antidepressant drug treatment for generalised anxiety, believing that drugs like imipramine were much less efficacious for anticipatory anxiety than for actual panic. Several studies that pre-dated the introduction of DSM-III found that tricyclic antidepressants such as amitriptyline and imipramine were efficacious in the treatment of anxiety symptoms (see Kahn et al, 1987), but some reviewers argued that the drugs were efficacious only in anxious patients who had co-existing panic disorder or depressive illness. This was difficult to dispute because studies tended to recruit patients with symptoms of both anxiety and depression. Recent large scale studies have addressed this topic.

Rickels et al (1993) conducted a study in which 230 patients who met DSM-III criteria for generalised anxiety disorder, but not major depression or panic disorder, were randomly allocated to treatment with imipramine, trazodone, diazepam or placebo. The average maximal daily doses were 143 $\mathrm{mg}$ for imipramine, $255 \mathrm{mg}$ for trazodone and $26 \mathrm{mg}$ for diazepam. Clinical assessments included Hamilton Anxiety Scale ratings conducted before treatment and over 8 weeks. Although treatment with diazepam was associated with the greatest reduction in ratings of anxiety over the first 2 weeks, 
it was treatment with imipramine that led to the greatest and most sustained improvement compared with placebo treatment. Patients who met the criteria for major depression were excluded from the study, but the authors assessed the relationship between the reduction in symptoms of anxiety and the number and/or severity of any depressive symptoms at the outset of treatment. There was no association overall between the reduction in anxiety associated with either antidepressant drug and the severity of depressive symptoms at the outset of treatment. However, patients with four complaints of depression (the maximum permitted for included patients) improved more with either antidepressant drug than with diazepam.

No published placebo-controlled studies of SSRIs were identified. Some reviewers have suggested that SSRIs are likely to be efficacious in generalised anxiety disorder and the study by Rocca et al in 1997 has been cited. These authors found paroxetine as efficacious as imipramine, but this finding is difficult to interpret because the maximum permissible dose of imipramine was $100 \mathrm{mg}$ /day and the average dose of imipramine was only $75 \mathrm{mg} /$ day.

The largest study on this topic included 377 patients recruited from several centres in the USA and treated with venlafaxine (Rickels et al, 2000). Patients met the DSM-IV criteria for generalised anxiety disorder, but not for major depressive disorder, and patients were excluded if they had a score on the Raskin Depression Scale (Raskin et al, 1969) of over three on any single item. Patients allocated to antidepressant drug treatment were prescribed the extended-release formulation of venlafaxine at a dose of 75, 150 or $225 \mathrm{mg} /$ day for up to 8 weeks. The patients allocated to the lowest dose were prescribed it from the outset of treatment, but the higher doses were not prescribed in full until after 2 weeks. The primary outcome measures included ratings on the Hamilton Anxiety Scale. The most substantial fall in anxiety was seen in the first 2 weeks, but anxiety scores continued to fall throughout the 8-week study. Each dose of venlafaxine led to a greater fall in anxiety scores than that seen with placebo, although the fall was most robust for the highest daily dose of venlafaxine.

\section{Discussion}

There is a growing body of evidence that antidepressant drug treatment can be efficacious in the treatment of anxiety disorders and not just when these are secondary to some other psychiatric condition such as depressive illness. Not all types of antidepressant drug treatment are of proven efficacy or of equal tolerability for a particular anxiety disorder, and there is now more evidence to inform selection. Nevertheless, there are still significant gaps in the evidence. Although the antipanic effect of imipramine was identified 40 years ago, it is still not known whether antidepressant drug treatment is more efficacious in spontaneous as opposed to situational panic attacks, in panic disorder with or without phobic avoidance, or whether certain somatic features of panic attacks respond better than others. A note of caution is appropriate for fluoxetine, the most popular branded antidepressant drug in the world. Although a large placebo-controlled study found fluoxetine (10-20 mg/day) no more efficacious than placebo in panic disorder, it is yet to be established whether this is explained by the specific pharmacology of the drug or the methodology of the study, for example, the prescribed dose range. It is also relevant to note that fluoxetine has not yet been shown to be efficacious in a placebocontrolled study of social phobia. It is disappointing

Box 2 Unresolved controversies about the pharmacotherapy of anxiety disorders

Recruitment

Can results taken from patients recruited by newspaper advertisements be generalised to psychiatric practice?

Design

Does the exclusion of patients with coexisting psychiatric conditions mean results cannot be generalised to psychiatric practice?

\section{Acute treatments}

Have acute-treatment studies been too short to meaningfully compare placebo with active treatment?

\section{Outcome}

Are the measures relevant to clinical practice? Would benefits be sustained after acute treatment?

Continuation treatment

For how long should pharmacotherapy be continued after initial response? Would the efficacy of psychological treatments be more enduring?

Cost

Would money spent on newer antidepressants be better invested in group psychological treatments provided by non-medical staff? 
to note that there is no good evidence of the efficacy of SSRIs in generalised anxiety disorder.

While the new evidence will be encouraging for sufferers and practitioners, there remain several unresolved controversies (see Box 2). Some studies depended on selecting patients whose anxiety symptoms are not associated with other psychiatric conditions, indeed many of the patients in these studies were recruited by newspaper advertisements and might never otherwise have come into psychiatric contact. Psychiatrists may need reassurance that this body of scientific evidence will be relevant for the patients they see in clinical practice. Unfortunately the extent to which these findings can be generalised to psychiatric practice has not yet been established. There are also strident critics of such research into the pharmacotherapy of anxiety disorders because it fails to acknowledge the significant numbers of patients who do not accept drug treatment. Also, the research fails to compare these drug treatments with psychological treatments that may take longer to work, but are more enduring in their benefits. Certainly there is a need for pragmatic studies that compare the pharmacotherapies of anxiety disorders with 'low tech' psychological treatments such as group CBT. This might not only establish whether or not either strategy alone or in combination is most efficacious in the longer term, but also inform sufferers of the most appropriate type of treatment.

\section{References}

American Psychiatric Association (1980) Diagnostic and Statistical Manual of Mental Disorders (3rd edn) (DSM-III). Washington, DC: APA.

- (1987) Diagnostic and Statistical Manual of Mental Disorders (3rd edn, revised) (DSM-III-R). Washington, DC: APA.

- (1994) Diagnostic and Statistical Manual of Mental Disorders (4th edn) (DSM-IV). Washington, DC: APA

Baldwin, D., Bobes, J., Stein, D. J., et al (1999) Paroxetine in social phobia/social anxiety disorder. Randomised, double-blind, placebo-controlled study. British Journal of Psychiatry, 175, 120-126.

Barlow, D. H., Gorman, J. M., Shear, M. K., et al (2000) Cognitive-behavioural therapy, impramine, or their combination for panic disorder. The Journal of the American Medical Association, 283, 2529-2536.

British Medical Association \& Royal Pharmaceutical Society of Greta Britain (2000) British National Formulary. London \& Wallingford: BMJ Books \& Pharmaceutical Press.

Charney, D. S., Woods, S. W., Goodman, W. K., et al (1986) Drug treatment of panic disorder: the comparative efficacy of imipramine, alprazolam, and trazodone. Journal of Clinical Psychiatry, 47, 580-586.

Cowen, P. J. (1997) Pharmacotherapy for anxiety disorders: drugs available. Advances in Psychiatric Treatment, 3, 6671 .

den Boer, J. A. \& Westenberg, H. G. (1988) Effect of serotonin and noradrenaline uptake inhibitors in panic disorder; a double-blind comparative study with fluvoxamine and maprotiline. International Clinical Psychopharmacology, 3, $59-74$.
- Bosker, F. J. \& Slaap, B. R. (2000) Serotonergic drugs in the treatment of depressive and anxiety disorders. Human Psychopharmacology, 15, 315-336.

El-Khayat, R. \& Baldwin, D. S. (1998) Antipsychotic drugs for non-psychotic patients: assessment of the benefit/risk ratio in generalised anxiety disorder. Journal of Psychopharmacology, 12, 323-329.

Fineberg, N. (1999) Evidence-based pharmacotherapy for obsessive-compulsive disorder. Advances in Psychiatric Treatment, 5, 357-365.

Hamilton, M. (1960) A rating scale for depression. Journal of Neurology, Neurosurgery and Psychiatry, 23, 56-62.

Kahn, R. J., McNair, D. M. \& Frankenthaler, L. M. (1987) Tricyclic treatment of generalized anxiety disorder. Journal of Affective Disorders, 13, 145-151.

Kruger, M. B. \& Dahl, A. A. (1999) The efficacy and safety of moclobemide compared to clomipramine in the treatment of panic disorder. European Archives of Psychiatry and Clinical Neuroscience, 249 (suppl. 1), S19-S24.

Loerch, B., Graf-Morgenstern, M., Hautzinger, M., et al (1999) Randomised placebo-controlled trial of moclobemide, cognitive-behavioural therapy and their combination in panic disorder with agoraphobia. British Journal of Psychiatry, 174, 205-212.

Lucrubier, Y., Bakker, A., Dunbar, G., et al (1997) A comparison of paroxetine, clomipramine and placebo in the treatment of panic disorder. Acta Psychiatrica Scandinavica, 95, 145-152.

Lydiard, R. B., Morton, W. A., Emmanuel, N. P., et al (1993) Preliminary report: placebo-controlled, double-blind study of the clincial and metabolic effects of desipramine in panic disorder. Psychopharmacology Bulletin, 29, 183-188.

Mavissakalian, M. R. \& Perel, J. M. (1999) Long-term maintenance and discontinuation of imipramine therapy in panic disorder with agoraphobia. Archives of General Psychiatry, 56, 821-827.

Michelson, D., Lydiard, R. B., Pollack, M. H., et al (1998) Outcome assessment and clinical improvement in panic disorder; evidence from a randomised controlled trial of fluoxetine and placebo. American Journal of Psychiatry, 155, 1570-1577.

--, Pollack, M., Lydiard, R. B., et al (1999) Continuing treatment of panic disorder after acute response: randomised, placebo-controlled trial with fluoxetine. British Journal of Psychiatry, 174, 213-218.

Modigh, K., Westberg, P. \& Eriksson, E. (1992) Superiority of clomipramine over imipramine in the treatment of panic disorder: a placebo-controlled trial. Journal of Clinical Psychopharmacology, 12, 251-261.

Noyes, R., Moroz, G., Davidson, J. R. T., et al (1997) Moclobemide and social phobia: a controlled doseresponse trial. Journal of Clinical Psychopharmacology, 17, 247-254

Nutt, D. \& Bell, C. (1997) Practical pharmacotherapy for anxiety. Advances in Psychiatric Treatment, 3, 79-85.

Raskin, A., Schulterbrant, J., Reatig, N., et al (1969) Replication of factors of psychopatholgoy in interview, word behavior, and self-report ratings of hospitalized depressives. Journal of Nervous and Mental Diseases, 148, 87-98.

Rickels, K., Downing, R., Schweizer, E., et al (1993) Antidepressants for the treatment of generalized anxiety disorder. Archives of General Psychiatry, 50, 884-895.

-, Pollack, M. H., Sheehan, D. V., et al (2000) Efficacy of extended-release venlafaxine in non-depressed oupatients with generalized anxiety disorder. American Journal of Psychiatry, 157, 968-974

Rocca, P., Fonzo, V., Scotta, M., et al (1997) Paroxetine efficacy in the treatment of generalized anxiety disorder. Acta Psychiatrica Scandanavica, 95, 444-450.

Simpson, H. B., Schneier, F. R., Campeas, R. B., et al (1998) Imipramine in the treatment of social phobia. Journal of Clinical Psychopharmacology, 18, 132-135.

Tiller, J. W. G., Bouwer, C. \& Behnke, K. (1999) Moclobemide and fluoxetine for panic disorder. European Archives of Psychiatry and Clinical Neuroscience, 249 (suppl. 1), S7-S10. 
Turner, S. (2000) Psychiatric help for survivors of torture. Advances in Psychiatric Treatment, 6, 295-303.

Tyrer, P. (1997) Pharmacotherapy for anxiety disorders: using the available drugs. Advances in Psychiatric Treatment, $3,72-78$.

Walker, J. R. \& Kjernisted, K. D. (2000) Fear: the impact and treatment of social phobia. Journal of Psychopharmacology, 14 (suppl. 1), S13-S23.

World Health Organization (1992) The ICD-10 Classification of Mental and Behavioural Disorders: Clinical Description and Diagnostic Guidelines. Geneva: WHO.

\section{Multiple choice questions}

1. There is good evidence of the efficacy of the following drugs in panic disorder:
a imipramine
b moclobemide
c trazodone
d fluoxetine
e paroxetine.

2. The following drugs are correctly paired with their minimum efficacious dose in panic disorder:
a imipramine: $25 \mathrm{mg} /$ day
b trazodone: $150 \mathrm{mg} /$ day
c fluoxetine: $10 \mathrm{mg} /$ day
d paroxetine: $40 \mathrm{mg} /$ day
e citalopram: $20-30 \mathrm{mg} /$ day.

3. There is good evidence of the efficacy of the following drugs in social phobia:
a imipramine
b phenelzine
c moclobemide
d fluoxetine
e paroxetine.

4. There is good evidence of the efficacy of the following drugs in generalised anxiety disorder:
a imipramine
b moclobemide
c fluoxetine
d citalopram
e venlafaxine.

5. In the treatment of panic disorder, there is no good evidence that:

a the selection of a tricyclic antidepressant drug has any significant effect on acute treatment outcome

b treatment with imipramine beyond 12 weeks is of any benefit

c patients without coexisting major depression respond to antidepressant drug treatment

$\mathrm{d}$ any psychological treatment apart from cognitive-behavioural therapy is of proven efficacy in acute treatment

e cognitive-behavioural therapy is of any greater efficacy than imipramine in preventing relapse after acute treatment.

\begin{tabular}{|c|c|c|c|c|}
\hline \multicolumn{5}{|c|}{ MCQ answers } \\
\hline \multirow{2}{*}{$\begin{array}{ll}1 & \\
\text { a } & T\end{array}$} & 2 & 3 & 4 & \multirow{2}{*}{$\begin{array}{ll}5 & \\
\text { a } & F\end{array}$} \\
\hline & a $F$ & a $F$ & a $\mathrm{T}$ & \\
\hline b $\mathbf{F}$ & b F & b $T$ & b F & b $F$ \\
\hline c $\quad F$ & c F & c $F$ & c $\mathbf{F}$ & c $F$ \\
\hline d F & d $\mathbf{T}$ & d F & d F & d F \\
\hline e $\mathbf{T}$ & e $\mathbf{T}$ & e $\mathbf{T}$ & e $\mathbf{T}$ & e $F$ \\
\hline
\end{tabular}

